\title{
Explicit Estimates for the Error Term in the Prime Number Theorem for Arithmetic Progressions
}

\author{
By Kevin S. McCurley
}

\begin{abstract}
We give explicit numerical estimates for the Chebyshev functions $\psi(x ; k, l)$ and $\boldsymbol{\theta}(x ; k, l)$ for certain nonexceptional moduli $k$. For values of $\varepsilon$ and $b$, a constant $c$ is tabulated such that $|\psi(x ; k, l)-x / \varphi(k)|<\varepsilon x / \varphi(k)$, provided $(k, l)=1, x \geqslant \exp \left(c \log ^{2} k\right)$, and $k \geqslant 10^{h}$. The methods are similar to those used by Rosser and Schoenfeld in the case $k=1$, but are based on explicit estimates of $N(T, \chi)$ and an explicit zero-free region for Dirichlet $L$-functions.
\end{abstract}

1. Introduction. Let $k$ and $l$ be positive integers. The Chebyshev prime counting functions $\psi(x ; k, l)$ and $\theta(x ; k, l)$ are defined by

$$
\theta(x ; k, l)=\sum_{\substack{p \leqslant x \\ p \equiv l(\bmod k)}} \log p, \quad \psi(x ; k, l)=\sum_{\substack{p^{\alpha} \leqslant x \\ p^{\alpha} \equiv l(\bmod k)}} \log p,
$$

where the sums extend over all primes $p$ and prime powers $p^{\alpha}$, respectively. The prime number theorem for arithmetic progressions is equivalent to the statement that

$$
\psi(x ; k, l)=x / \varphi(k)+o(x), \quad x \rightarrow \infty,
$$

if $k$ and $l$ are fixed relatively prime integers. An alternative statement is that for any positive $\varepsilon$ there exists $x_{0}=x_{0}(k, l, \varepsilon)$ such that

$$
|\psi(x ; k, l)-x / \varphi(k)|<\varepsilon x / \varphi(k), \quad x \geqslant x_{0} .
$$

The purpose of this paper is to give explicit numerical estimates for $x_{0}(k, l, \varepsilon)$ for some values of $k$ and $\varepsilon$.

The case $k=1$ or 2 has been investigated in a series of papers by J. B. Rosser and L. Schoenfeld. The methods used in this paper are similar to those used by Rosser and Schoenfeld, and we shall make frequent reference to their work.

The size of the error term in the prime number theorem depends on the location of zeros of the Riemann zeta function $\zeta(s)$. The estimates of $\psi(x ; 1,1)$ in $[10]$ and [11] are based on the computation of 3,502,500 zeros of $\zeta(s)$ and a zero-free region for $\zeta(s)$ of the type originally proved by de la Vallee Poussin. A similar situation exists in the case of the prime number theorem for arithmetic progressions, where the size of $x_{0}(k, l, \varepsilon)$ depends on the location of zeros of Dirichlet $L$-functions formed with characters modulo $k$. In the case of a fixed modulus $k$ we can make use

Received June 21, 1982.

1980 Mathematics Subject Classification. Primary 10H20, $10 \mathrm{H} 08$.

(1)1984 American Mathematical Society $0025-5718 / 84 \$ 1.00+\$ .25$ per page 
of computational information concerning the zeros of $L$-functions modulo $k$ in the same way that Rosser and Schoenfeld used information concerning the zeros of $\zeta(s)$. In the estimation of $x_{0}(k, l, \varepsilon)$ as $k$ tends to infinity, we can no longer derive significant benefit from the mere computation of zeros, since it is no longer a finite computational problem to compute enough zeros. In this case we can base our estimates on the following explicit zero-free region.

Let $R=9.645908801$ and $\mathcal{E}_{k}(s)=\prod_{x \bmod k} L(s, \chi)$.

THEOREM 1.1. There exists at most a single zero of $\mathcal{L}_{k}(s)$ in the region $\{s=\sigma+i t:$ $\sigma \geqslant 1-1 /[R \log \max \{k, k|t|, 10\}]\}$. The only possible zero in this region is a simple real zero arising from an L-function formed with a real nonprincipal character modulo K.

If $k$ is an integer for which there exists a real zero of $E_{k}(s)$ with $\beta>1-$ $1 /(R \log k)$, then we shall refer to $k$ as an exceptional modulus. A proof of Theorem 1.1 appears in [5], as well as a further result concerning exceptional moduli.

TABLE 1

\begin{tabular}{|c|c|c|c|c|c|c|c|c|c|c|}
\hline$b$ & 1 & .5 & .2 & .1 & .05 & .01 & .005 & .001 & . & $.(0()) !) \mathrm{i}$ \\
\hline 1 & 34.13 & 41.01 & 53.23 & 65.28 & 79.94 & 124.3 & 147.2 & 208.3 & 313.3 & $432^{2} .5$ \\
\hline 2 & 20.62 & 23.35 & 27.98 & 32.37 & 37.55 & 52.25 & 59.53 & 78.34 & $109 .:$ & i幺6. \\
\hline 3 & 16.85 & 18.51 & 21.29 & 23.88 & 26.84 & 34.92 & 38.82 & 48.74 & 54.88 & 83.19 \\
\hline 4 & 15.08 & 16.28 & 18.26 & 20.05 & 22.08 & 27.48 & 30.04 & 36.49 & 46.30 & 58.36 \\
\hline 5 & 14.04 & 14.98 & 16.51 & 17.88 & $19 . \therefore 1$ & 23.41 & 25.88 & $: 4.97$ & 37.34 & $\therefore 5.5$ \\
\hline 6 & 13.36 & 14.12 & 13.37 & 16.47 & 17.69 & 20.83 & 22.33 & 25.94 & 31.62 & 37.34 \\
\hline$i$ & 12.86 & 13.52 & 14.58 & 15.50 & 16.49 & 19.09 & 20.28 & 23.23 & 27.81 & 32.78 \\
\hline d & 12.49 & 13.07 & 13.98 & 14. in & 15.62 & 17.81 & 18.82 & 21.29 & 25.11 & 29.23 \\
\hline 9 & 12.20 & 12.73 & 13.52 & 14.21 & 14.04 & 16.86 & 17.72 & 19.85 & 23.09 & 25.58 \\
\hline 10 & 11.98 & 12.44 & 13.15 & 13.76 & 14.43 & 16.10 & 16.87 & 18.71 & 21.54 & 24.57 \\
\hline 11 & 11.79 & 12.20 & 12.85 & 13.38 & 13.99 & 15.47 & 16.15 & 17.81 & 20.30 & 22.95 \\
\hline 12 & 11.64 & 12.03 & 12.60 & 13.09 & 13.62 & 14.99 & 15.58 & 17.08 & 10.31 & 21.57 \\
\hline 13 & 11.50 & 11.84 & 12.37 & 12.83 & 13.32 & 14.55 & i5.10 & 16.44 & 18.48 & 20.60 \\
\hline 14 & 11.39 & 11.72 & 12.19 & 12.62 & 13.08 & 14.20 & 14.71 & 15.93 & 17.76 & 19.72 \\
\hline 15 & 11.29 & 11.58 & 12.03 & 12.42 & 12.85 & 13.88 & 14.35 & 15.48 & 17.17 & 18.96 \\
\hline 20 & 10.93 & 11.14 & $1 ! .48$ & 11.76 & 12.08 & 12.81 & 13.10 & 13.97 & 15.15 & 16.41 \\
\hline 25 & 10.67 & 10.88 & 11.14 & 11.36 & 11.60 & i 2.20 & 12.45 & $13.0 \%$ & 14.00 & 14.95 \\
\hline 30 & 10.55 & 10.69 & 10.91 & 11.09 & 11.30 & 11.78 & 11.99 & 12.51 & 13.25 & 14.01 \\
\hline 35 & $10 . \dot{44}$ & 10.55 & 10.74 & 10.90 & 11.08 & $11.4 ?$ & 11.65 & 12.08 & 12.72 & 13.37 \\
\hline 40 & 10.35 & 10.45 & 10.62 & 10.76 & 10.90 & 11.25 & $11 . \therefore 1$ & 11.79 & 12.32 & 12.89 \\
\hline 45 & 10.29 & 10.39 & 10.52 & 10.64 & 10.78 & 11.08 & 11.23 & 11.54 & 12.02 & 12.51 \\
\hline 50 & 10.24 & 10.33 & 10.45 & 10.56 & 10.66 & 10.95 & 11.06 & 11.37 & 11.78 & 12.22 \\
\hline 60 & 10.15 & 10.21 & 10.32 & 10.43 & 10.52 & 10.74 & 10.84 & 11.08 & 11.42 & 11.78 \\
\hline 70 & 10.08 & 10.14 & 10.23 & 10.32 & 10.40 & 10.59 & 10.68 & 10.89 & 11.17 & 11.47 \\
\hline 80 & 10.04 & 10.08 & 10.18 & 10.23 & 10.31 & 10.48 & 10.56 & 10.73 & 10.98 & $1: .25$ \\
\hline 90 & 10.00 & 10.04 & $: 0.11$ & 10.18 & 10.23 & 10.41 & 10.45 & 10.61 & 10.85 & 11.08 \\
\hline 100 & 9.96 & 10.01 & 10.07 & 10.13 & 10.18 & 10.32 & 10.39 & 10.54 & 10.72 & ifj.93 \\
\hline
\end{tabular}


The main result of this paper is the following:

TheOREM 1.2. Let $k$ be a nonexceptional modulus, and let $(k, l)=1$. For various values of $\varepsilon$ and $b$, Table 1 gives values of $c$ such that

$$
\left|\psi(x ; k, l)-\frac{x}{\varphi(k)}\right|<\frac{\varepsilon x}{\varphi(k)} \text { and }\left|\theta(x ; k, l)-\frac{x}{\varphi(k)}\right|<\frac{\varepsilon x}{\varphi(k)},
$$

provided that $k \geqslant 10^{b}$ and $x \geqslant \exp \left(c \log ^{2} k\right)$.

For any given values of $\varepsilon$ and $b$ the methods of this paper will yield a value of $c$, but the methods are limited by the requirement that $c>R$. The methods could also be used to calculate an explicit constant $A$ with the property that

$$
\frac{\varphi(k)}{x}\left|\psi(x ; k, l)-\frac{x}{\varphi(k)}\right|<A k \sqrt{\frac{\log x}{R}} \exp \left(-\sqrt{\frac{\log x}{R}}\right),
$$

provided $x>\exp \left(R \log ^{2} k\right)$ and $k$ is not exceptional. In the interests of brevity this will be deferred to a later paper. Later papers will also deal with the case $k=3$ and implications of the generalized Riemann hypothesis.

2. Estimates of $N(T, \chi)$. Throughout this paper $\chi$ will be a Dirichlet character modulo $k$, and $\chi_{1}$ modulo $k_{1}$ will be the primitive character which induces $\chi$. We write $\chi_{0}$ for the principal character, and in this case we take $k_{1}=1$ and $\chi_{1}=1$. Note that

$$
L(s, \chi)=L\left(s, \chi_{1}\right) \prod_{p \nmid h}\left(1-\chi_{1}(p) p^{\cdots}\right)
$$

Define $N(T, \chi)=N\left(T, \chi_{1}\right)$ as the number of zeros $\rho=\beta+i \gamma$ of $L(s, \chi)$ with $0<\beta<1$ and $|\gamma| \leqslant T$. The main result of this section is the following.

THEOREM 2.1. Let $T \geqslant 1$ and $\chi$ be a primitive nonprincipal character modulo $k$. If $0<\eta \leqslant \frac{1}{2}$, then

$$
\left|N(T, \chi)-\frac{T}{\pi} \log \frac{k T}{2 \pi e}\right|<C_{1} \log k T+C_{2}
$$

where

$$
\begin{gathered}
C_{1}=\frac{1+2 \eta}{\pi \log 2}, \\
C_{2}=.3058-.268 \eta+\frac{4 \log \zeta(1+\eta)}{\log 2}-\frac{2 \log \zeta(2+2 \eta)}{\log 2} \\
+\frac{2}{\pi} \log \zeta\left(\frac{3}{2}+2 \eta\right) .
\end{gathered}
$$

Proof. The method of proof is essentially due to Backlund [2], with refinements due to Rosser [9] and the author. Assuming that $\pm T$ does not coincide with the ordinate of a zero, consider the rectangle $R$ with vertices at $\sigma_{1}-i T, \sigma_{1}+i T$, $1-\sigma_{1}+i T$, and $1-\sigma_{1}-i T$, where $\sigma_{1}>1$. Then we have

$$
N(T, \chi)=\frac{1}{2 \pi} \Delta_{R} \arg \xi(s, \chi),
$$


where

$$
\xi(s, \chi)=\left(\frac{k}{\pi}\right)^{(s+a) / 2} \Gamma\left(\frac{s+a}{2}\right) L(s, \chi), \quad a=(1-\chi(-1)) / 2 .
$$

Let $e$ denote the portion of the contour in $\sigma \geqslant \frac{1}{2}$. From the functional equation of $\xi(s, \chi)$ and (2.6) we obtain

$$
\begin{aligned}
\Delta_{R} \arg \xi(s, \chi) & =2 \Delta_{\circlearrowright} \arg \xi(s, \chi) \\
& =2\left[\Delta_{\circlearrowright} \arg \left(\frac{k}{\pi}\right)^{(s+a) / 2}+\Delta_{\circlearrowright} \arg \Gamma\left(\frac{s+a}{2}\right)+\Delta_{c^{\prime}} \arg L(s, \chi)\right] \\
& =2 T \log \frac{k}{\pi}+4 \operatorname{Im} \log \Gamma\left(\frac{1}{4}+\frac{a}{2}+i \frac{T}{2}\right)+2 \Delta_{\circlearrowright} \arg L(s, \chi) .
\end{aligned}
$$

We shall apply Stirling's formula in the form

$$
\log \Gamma(z)=\left(z-\frac{1}{2}\right) \log z-z+\frac{1}{2} \log 2 \pi+\frac{\theta}{6|z|},
$$

where $|\theta| \leqslant 1$ and $|\arg z| \leqslant \pi / 2$. This error term is well known and appears in Olver [6, p. 294]. Hence

$$
\begin{aligned}
\operatorname{Im} \log \Gamma\left(\frac{1}{4}+\frac{a}{2}+i \frac{T}{2}\right)= & \frac{T}{2} \log \frac{T}{2 e}+\frac{T}{4} \log \left[1+\left(\frac{2 a+1}{2 T}\right)^{2}\right] \\
& +\frac{2 a-1}{4} \tan ^{-1}\left[\frac{2 T}{1+2 a}\right]+\frac{\theta}{3\left|\frac{1}{2}+a+i T\right|}
\end{aligned}
$$

Denote the last three terms by $f_{1}(T), f_{2}(T)$ and $f_{3}(T)$. If $a=0$, then $f_{1}$ and $f_{2}$ are decreasing for $T \geqslant 1$, so that

$$
\begin{aligned}
\left|f_{1}+f_{2}+f_{3}\right| & \leqslant\left|f_{1}+f_{2}\right|+\frac{1}{3 \sqrt{1.25}} \\
& \leqslant \max \left\{\left|f_{1}(1)+f_{2}(1)\right|,\left|f_{1}(\infty)+f_{2}(\infty)\right|\right\}+.2982<.6909
\end{aligned}
$$

If $a=1$, then $f_{1}$ and $f_{2}$ are positive, $f_{1}$ is decreasing, and $f_{2}$ is increasing. The maximum value of $f_{1}+f_{2}$ occurs between $T=1.64$ and $T=1.65$, so that

$$
\left|f_{1}+f_{2}+f_{3}\right|<f_{1}(1.64)+f_{2}(1.65)+\frac{1}{3 \sqrt{3.25}}<.6425
$$

It follows from (2.5), (2.7) and (2.8) that

$$
N(T, \chi)=\frac{1}{\pi}\left[T \log \frac{k T}{2 \pi e}+1.3818 \theta+\Delta_{\mathcal{C}} \arg L(s, \chi)\right] .
$$

It remains to estimate $\Delta_{\mathcal{C}}$ arg $L(s, \chi)$. We divide $\mathcal{C}_{\text {into }} 3$ pieces $\mathcal{C}_{1}, \bigodot_{2}$, and $\mathcal{C}_{3}$ as follows:

$$
\begin{array}{lll}
e_{1}: \frac{1}{2}-i T & \text { to } & \sigma_{1}-i T, \\
e_{2}: \sigma_{1}-i T & \text { to } & \sigma_{1}+i T, \\
e_{3}: \sigma_{1}+i T & \text { to } & \frac{1}{2}+i T .
\end{array}
$$

We first estimate $\Delta_{e_{3}} \arg L(s, \chi)$. In view of the fact that $L(\bar{s}, \chi)=\overline{L(s, \bar{\chi})}$, an upper bound for the change in argument on $C_{3}$ will also serve as an upper bound on 
$e_{1}$, provided the bound is valid for any primitive nonprincipal $\chi$ modulo $k$.

Let $N$ be a positive integer, and define

$$
f(s)=\frac{1}{2}\left[L(s+i T, \chi)^{N}+L(s-i T, \bar{\chi})^{N}\right] .
$$

Note that

$$
f(\sigma)=\operatorname{Re} L(\sigma+i T, \chi)^{N}
$$

if $\sigma$ is real. Suppose $f(\sigma)$ has $n$ real zeros in the interval $\frac{1}{2} \leqslant \sigma \leqslant \sigma_{1}$. These zeros partition the interval into $n+1$ subintervals, and on each subinterval the quantity $\arg L(\sigma+i T, \chi)^{N}$ can change by at most $\pi$, since $\operatorname{Re} L(\sigma+i T, \chi)^{N}$ is nonzero on the interior of each subinterval. It follows that

$$
\left|\Delta_{\Theta_{3}} \arg L(s, \chi)\right|=\frac{1}{N}\left|\Delta_{e_{3}} \arg L(s, \chi)^{N}\right| \leqslant \frac{(n+1) \pi}{N} .
$$

We now estimate $n$ from above. Let $0<\eta \leqslant \frac{1}{2}$, and define $\sigma_{1}=\frac{3}{2}+2 \eta$ and $\sigma_{0}=1+\eta$. It follows from Jensen's theorem that

$$
n \log 2 \leqslant \frac{1}{2 \pi} \int_{-\pi / 2}^{3 \pi / 2} \log \left|f\left(\sigma_{0}+(1+2 \eta) e^{i \theta}\right)\right| d \theta-\log \left|f\left(\sigma_{0}\right)\right| .
$$

In order to estimate $|f(s)|$ we appeal to a result of Rademacher [8]. He proved that if $-\eta \leqslant \sigma \leqslant 1+\eta$, then

$$
|L(s, \chi)| \leqslant\left(\frac{k|s+1|}{2 \pi}\right)^{(1+\eta-\sigma) / 2} \zeta(1+\eta) .
$$

It follows that

$$
\begin{aligned}
& \frac{1}{2 \pi} \int_{\pi / 2}^{3 \pi / 2} \log \left|f\left(\sigma_{0}+(1+2 \eta) e^{i \theta}\right)\right| d \theta \\
& \leqslant \frac{-N}{2 \pi} \int_{\pi / 2}^{3 \pi / 2} \frac{1}{2}(1+2 \eta) \cos \theta \log \left(\frac{k\left(\sqrt{T^{2}+(2+\eta)^{2}}+1+2 \eta\right)}{2 \pi}\right) d \theta \\
& \quad+\frac{N}{2} \log \zeta(1+\eta) \\
& \leqslant \frac{N}{2 \pi}(1+2 \eta) \log (.74685 k T)+\frac{N}{2} \log \zeta(1+\eta)
\end{aligned}
$$

since $T \geqslant 1$ and $\eta \leqslant \frac{1}{2}$.

If $\sigma \geqslant 1+\eta$, then we use the trivial estimate

$$
|f(s)| \leqslant \zeta(1+\eta)^{N},
$$

and it follows from (2.11) and (2.12) that

(2.13) $n \log 2 \leqslant \frac{N(1+2 \eta)}{2 \pi} \log (.74685 k T)+N \log \zeta(1+\eta)-\log \left|f\left(\sigma_{0}\right)\right|$.

Now write $L\left(\sigma_{0}+i T, \chi\right)=r e^{i \varphi}$. We choose a sequence of $N$ 's tending to infinity such that $N \varphi$ tends to 0 modulo $2 \pi$. It follows that

$$
\lim _{N \rightarrow \infty} \frac{f\left(\sigma_{0}\right)}{\left|L\left(\sigma_{0}+i T, \chi\right)\right|^{N}}=1 .
$$


Note that for $\sigma>1$ we have

$$
|L(s, \chi)|=\prod_{p}\left|1-\chi(p) p^{-s}\right|^{-1} \geqslant \prod_{p}\left(1+\frac{1}{p^{\sigma}}\right)^{-1}=\frac{\zeta(2 \sigma)}{\zeta(\sigma)} .
$$

Hence from (2.10), (2.13), and (2.14) we obtain

$$
\begin{aligned}
\left|\Delta_{c_{3}} \arg L(s, \chi)\right| \leqslant & \frac{1+2 \eta}{2 \log 2} \log (.74685 k T) \\
& +\frac{2 \pi \log \zeta(1+\eta)}{\log 2}-\frac{\pi \log \zeta(2+2 \eta)}{\log 2} .
\end{aligned}
$$

Finally we estimate the change along $e_{2}$. If $\sigma>1$, then

$$
|\arg L(s, \chi)| \leqslant|\log L(s, \chi)| \leqslant \log \zeta(\sigma) .
$$

Hence

$$
\left|\Delta_{c:} \arg L(s, \chi)\right| \leqslant 2 \log \zeta\left(\frac{3}{2}+2 \eta\right) \text {. }
$$

The result then follows from (2.9) and (2.15).

Theorem 2.1 may be stated as well for imprimitive or principal characters. Henceforth we shall abbreviate $N(T, \chi)$ as $N(T)$, and furthermore we use

$$
\begin{aligned}
& F(T)=\frac{T}{\pi} \log \frac{k T}{2 \pi e}, \quad F_{1}(T)=\frac{T}{\pi} \log \frac{k_{1} T}{2 \pi e}, \\
& R(T)=C_{1} \log k T+C_{2}, \quad R_{1}(T)=C_{1} \log k_{1} T+C_{2} .
\end{aligned}
$$

Corollary 2.2. If $T \geqslant 1$ and $C_{1}$ and $C_{2}$ are as in Theorem 2.1, then

$$
\left|N(T)-F_{1}(T)\right|<R_{1}(T) .
$$

Proof. If $\chi$ is nonprincipal this follow immediately from Theorem 2.1. since $N\left(T, \chi_{1}\right)=N(T, \chi)$. If $\chi=\chi_{0}$ is the principal character, then we appeal to a result of Rosser [9], who proved that (in our notation)

$$
\begin{aligned}
& \left|N\left(T, x_{0}\right)-\frac{T}{\pi} \log \frac{T}{2 \pi e}\right| \\
& \quad< \begin{cases}3.75, & 0<T \leqslant 280 \\
5.75, & 0<T \leqslant 1467 \\
.274 \log T+.886 \log \log T+4.926, & 2 \leqslant T .\end{cases}
\end{aligned}
$$

If $\sigma>1$, note that

$$
2 \log \zeta(\sigma)-\log \zeta(2 \sigma)=\sum_{p} \log \left(\frac{p^{\sigma}+1}{p^{\sigma}-1}\right)
$$

is decreasing in $\sigma$. It follows that $C_{2}$ is decreasing in $\eta$, and

$$
C_{2}>5.365 \text {. }
$$

If $1 \leqslant T \leqslant 280$ or $280 \leqslant T \leqslant 1467$, the result follows immediately from (2.17) and (2.18). If $T \geqslant 1467$, then by (2.17) and (2.18) it suffices to prove that

$$
\left(\frac{1}{\pi \log 2}-.274\right) \log T-.886 \log \log T+.439>0 .
$$

The left side of (2.19) is increasing in $T$ for $T \geqslant 1467$, and is positive for $T=1467$. 
3. Bounds for $\psi(x ; k, l)$. Let $k$ and $l$ be positive integers with $(k, l)=1$. Our method of estimation for $\psi(x ; k, l)$ is based on an "explicit formula" for certain integral averages of $\psi(x ; k, l)$. This is the method used by Rosser [9] in the case $k=1$, and reduces the problem to that of estimating certain sums involving zeros of Dirichlet $L$-functions.

Before we state the explicit formula we require some notation. If $\chi$ is a Dirichlet character modulo $k$, we use $z(\chi)$ to represent the set of zeros $\rho=\beta+i \gamma$ of $L(s, \chi)$ with $\beta \geqslant 0$ and $\rho \neq 0$. Since $\chi_{1}$ is the associated primitive character, $z\left(\chi_{1}\right)$ is the subset of $z(\chi)$ consisting of the zeros with $\beta>0$. We use $b(\chi)$ for the constant term in the Laurent expansion of $L^{\prime}(s, \chi) / L(s, \chi)$ about $0, c(\chi)$ for the constant term in the expansion about -1 , and $m(\chi)$ for the order of the zero of $L(s, \chi)$ at $s=0$. Note that

$$
0 \leqslant m(\chi) \leqslant \omega(k) \leqslant \frac{\log k}{\log 2},
$$

where $\omega(k)$ is the number of distinct prime factors of $k$. Unless otherwise indicated, a sum over $\chi$ is to be interpreted as a sum over all characters modulo $k$.

LemMa 3.1. Let $\psi_{1}(x ; k, l)=\int_{1}^{x} \psi(t ; k, l) d t$, where $x>1$. Then

$$
\begin{aligned}
\psi_{1}(x ; k, l)= & \frac{x^{2}}{2 \varphi(k)}-\frac{1}{\varphi(k)} \sum_{\chi} \bar{\chi}(l) \sum_{\rho \in z(\chi)} \frac{x^{\rho+1}}{\rho(\rho+1)}+g(x) \\
& +d_{1} x+d_{2} x \log x+d_{3} \log x+d_{4},
\end{aligned}
$$

where

$$
\begin{aligned}
g(x) & =-\frac{1}{\varphi(k)} \sum_{\chi} \bar{\chi}(l) \sum_{n=1}^{\infty} \frac{x^{-2 n+1-a}}{2 n(2 n-1+2 a)} \\
a & =(1-\chi(-1)) / 2 \\
d_{1} & =\frac{1}{\varphi(k)} \sum_{\chi} \bar{\chi}(l)[m(\chi)-b(\chi)] \\
d_{2} & =\frac{-1}{\varphi(k)} \sum_{\chi} \bar{\chi}(l) m(\chi), \\
d_{3} & =\frac{1}{\varphi(k)} \sum_{x(-1)=-1} \bar{\chi}(l), \\
d_{4} & =\frac{1}{\varphi(k)} \sum_{x(-1)=1} \bar{\chi}(l) \frac{L^{\prime}}{L}(-1, \chi)+\frac{1}{\varphi(k)} \sum_{x(-1)=-1} \bar{\chi}(l)[c(\chi)+1] .
\end{aligned}
$$

Proof. A "smoothed" Perron inversion formula gives

$$
\psi_{1}(x ; k, l)=\frac{-1}{\varphi(k)} \sum_{\chi} \bar{\chi}(l) \frac{1}{2 \pi} \int_{2-i \infty}^{2+i \infty} \frac{x^{s+1}}{s(s+1)} \frac{L^{\prime}}{L}(s, \chi) d s .
$$

The remainder of the proof involves an application of the residue theorem to express the contour integral as a sum of residues. The details justifying this appear in Ingham [4, pp. 68-74], and Prachar [7, pp. 224-228]. 
For $x>1$, define

$$
E(x)=\psi(x ; k, l)-x / \varphi(k),
$$

and for $m$ a positive integer, $x+m h>1$, define

$$
E_{m}(x, h)=\int_{0}^{h} \cdots \int_{0}^{h} E\left(x+y_{1}+\cdots+y_{m}\right) d y_{1} \cdots d y_{m} .
$$

Further let

$$
f(x)=\frac{1}{\varphi(k)} \sum_{x} \bar{\chi}(l) \sum_{n=1}^{\infty} \frac{x^{-2 n+a}}{2 n-a}+d_{2} \log x+d_{1}+d_{2} .
$$

LEMMA 3.2. If $|h|<(x-1) / m$, then

$$
\begin{aligned}
& E_{m}(x, h) \\
& =\frac{1}{\varphi(k)} \sum_{x} \bar{\chi}(l) \sum_{\rho \in z(x)} \frac{1}{\rho(\rho+1) \cdots(\rho+m)} \sum_{j=0}^{m}(-1)^{m+j+1}\left(\begin{array}{c}
m \\
j
\end{array}\right)(x+j h)^{\rho+m} \\
& \quad+\int_{0}^{h} \cdots \int_{0}^{h} f\left(x+y_{1}+\cdots+y_{m}\right) d y_{1} \cdots d y_{m} .
\end{aligned}
$$

Proof. We use induction on $m$. If $m=1$, it follows from (3.8), (3.9), and Lemma 3.1 that

$$
\begin{aligned}
E_{1}(x, h)= & \int_{0}^{h} E(x+y) d y \\
= & \frac{1}{\varphi(k)} \sum_{x} \bar{\chi}(l) \sum_{\rho \in z(x)} \frac{x^{\rho+1}-(x+h)^{\rho+1}}{\rho(\rho+1)}+d_{1} h \\
& +d_{2}(x+h) \log (x+h)-d_{2} x \log x+d_{3} \log (x+h) \\
& -d_{3} \log x+g(x+h)-g(x) .
\end{aligned}
$$

The result then follows for $m=1$ from (3.3), (3.4), (3.5), (3.6), and (3.10).

If $m>1$, we have

$$
\begin{aligned}
E_{m}(x, h)= & \int_{0}^{h} E_{m-1}\left(x+y_{m}, h\right) d y_{m} \\
= & \frac{1}{\varphi(k)} \sum_{x} \bar{\chi}(l) \sum_{\rho \in z(x)} \frac{1}{\rho(\rho+1) \cdots(\rho+m-1)} \\
& \times \sum_{j=0}^{m-1}(-1)^{m+j}\left(\begin{array}{c}
m-1 \\
j
\end{array}\right) \int_{0}^{h}\left(x+y_{m}+j h\right)^{\rho+m-1} d y_{m} \\
& +\int_{0}^{h} \cdots \int_{0}^{h} f\left(x+y_{1}+\cdots+y_{m}\right) d y_{1} \cdots d y_{m},
\end{aligned}
$$


the term-by-term integration being justified by the fact that $\sum_{\rho \in z(x)} 1 /|\rho(\rho+1)|$ converges. The sum on $j$ may be written as

$$
\begin{aligned}
\frac{1}{\rho+m} \sum_{j=0}^{m-1}(-1)^{m+j+1}\left(\begin{array}{c}
m-1 \\
j
\end{array}\right)(x+j h)^{\rho+m} \\
+\frac{1}{\rho+m} \sum_{i=1}^{m}(-1)^{m+i+1}\left(\begin{array}{c}
m-1 \\
i-1
\end{array}\right)(x+i h)^{\rho+m} \\
=\frac{1}{\rho+m} \sum_{j=0}^{m}(-1)^{m+j+1}\left(\begin{array}{c}
m \\
j
\end{array}\right)(x+j h)^{\rho+m}
\end{aligned}
$$

and this completes the proof.

LEMMA 3.3. If $0<h<(x-1) / m$, then there exists a $z$ such that $0 \leqslant z \leqslant m h$ and

$$
E(x+z) \leqslant \frac{E_{m}(x, h)}{h^{m}}+\frac{m h}{2 \varphi(k)}-\frac{z}{\varphi(k)} .
$$

If $0<-h<(x-1) / m$, then there exists a $z$ such that $m h \leqslant z \leqslant 0$ and

$$
E(x+z) \geqslant \frac{E_{m}(x, h)}{h^{m}}+\frac{m h}{2 \varphi(k)}-\frac{z}{\varphi(k)} .
$$

Proof. Let $G(t)=E(x+t)+t / \varphi(k)$. If $h>0$, then clearly there exists a $z$ such that $0 \leqslant z \leqslant m h$ and

$$
G(z) \leqslant \frac{1}{h^{m}} \int_{0}^{h} \cdots \int_{0}^{h} G\left(y_{1}+\cdots+y_{m}\right) d y_{1} \cdots d y_{m},
$$

and this proves the first part. If $h<0$, then there exists a $z$ such that $m h \leqslant z \leqslant 0$ and

$$
G(z) \geqslant \frac{1}{(-h)^{m}} \int_{h}^{0} \cdots \int_{h}^{0} G\left(y_{1}+\cdots+y_{m}\right) d y_{1} \cdots d y_{m} .
$$

LEMMA 3.4. If $0<\delta<(x-1) /(m x)$, then

$$
\frac{\varphi(k) E_{m}(x,-\delta x)}{(-\delta)^{m} x^{m+1}}-\frac{m \delta}{2} \leqslant \frac{\varphi(k)}{x} \psi(x ; k, l)-1 \leqslant \frac{\varphi(k) E_{m}(x, \delta x)}{\delta^{m} x^{m+1}}+\frac{m \delta}{2} \text {. }
$$

Proof. In Lemma 3.3 we put $h=\delta x$, and it follows that there exists a $z>0$ with

$$
\psi(x+z ; k, l) \leqslant \frac{x}{\varphi(k)}+\frac{E_{m}(x, \delta x)}{(\delta x)^{m}}+\frac{m \delta x}{2 \varphi(k)},
$$

but $\psi(x ; k, l) \leqslant \psi(x+z ; k, l)$, so that this proves the upper bound. The lower bound is proved with $h=-\delta x$.

This reduces the problem to the estimation of $\left|E_{m}(x, \pm \delta x)\right|$, for which we require a lemma.

LEMMA 3.5. If $d_{1}$ and $d_{2}$ are defined by (3.4) and (3.5), and $k$ is not exceptional, then

$$
\left|d_{1}+d_{2}\right| \leqslant \frac{k}{4} \log k+C_{3} \log ^{2} k+C_{4} \log k+C_{5},
$$


where

$$
\begin{aligned}
& C_{3}=11 C_{1}+4, \\
& C_{4}=11 C_{2}+2 C_{1}-8 \\
& C_{5}=C_{1}+2 C_{2}-2 .
\end{aligned}
$$

Proof. From (3.4) and (3.5) we obtain

$$
d_{1}+d_{2}=\frac{-1}{\varphi(k)} \sum_{\chi} \bar{\chi}(l) b(\chi)
$$

hence

$$
\left|d_{1}+d_{2}\right| \leqslant \max _{x}|b(\chi)| .
$$

If $\chi_{0}$ is the principal character modulo $k$, then

$$
\frac{L^{\prime}}{L}\left(s, x_{0}\right)=\frac{\zeta^{\prime}}{\zeta}(s)+\sum_{p \mid k} \frac{\log p}{p^{s}-1}
$$

and it follows that

$$
b\left(x_{0}\right)=\log 2 \pi-\frac{1}{2} \sum_{p \mid k} \log p .
$$

Hence we have trivially

$$
\left|b\left(x_{0}\right)\right| \leqslant \log 2 \pi+\frac{1}{2} \log k \leqslant 4 \log ^{2} k,
$$

and the result follows from (2.18).

If $\chi$ is nonprincipal, then from (2.1) we obtain

If $\chi_{1}(p) \neq 1$, note that

$$
b(x)=b\left(x_{1}\right)-\frac{1}{2} \sum_{\substack{p \mid k \\ x_{1}(p)=1}} \log p+\sum_{\substack{p \mid k \\ x_{1}(p)=1}} \frac{x_{1}(p) \log p}{1-x_{1}(p)} .
$$

hence

$$
\left|1-x_{1}(p)\right| \geqslant\left|1-\exp \left(\frac{2 \pi i}{\varphi\left(k_{1}\right)}\right)\right| \geqslant \frac{4}{k}
$$

$$
|b(x)| \leqslant\left|b\left(x_{1}\right)\right|+\sum_{p \mid k} \log p \max \left\{\frac{1}{2} \cdot \frac{k}{4}\right\} \leqslant\left|b\left(x_{1}\right)\right|+\frac{k}{4} \log k .
$$

From Davenport [3, p. 85] we have

$$
\frac{L^{\prime}}{L}\left(s, x_{1}\right)=\frac{1}{2} \log \frac{\pi}{k_{1}}-\frac{1}{2} \frac{\Gamma^{\prime}}{\Gamma}\left(\frac{s+a}{2}\right)+B\left(\chi_{1}\right)+\sum_{\rho \in z\left(\chi_{1}\right)}\left(\frac{1}{s-\rho}+\frac{1}{\rho}\right) .
$$

If we subtract the same expression with $s$ replaced by 2 , we obtain

$$
b\left(x_{1}\right)=\frac{L^{\prime}}{L}\left(2, x_{1}\right)+a-\sum_{\rho \in z\left(x_{1}\right)} \frac{2}{\rho(2-\rho)},
$$

and it follows from (3.15) that

$$
|b(x)| \leqslant\left|\frac{\zeta^{\prime}}{\zeta}(2)\right|+1+\sum_{\rho \in z\left(x_{1}\right)} \frac{2}{|\rho(2-\rho)|}+\frac{k}{4} \log k .
$$


It remains to estimate the sum on $\rho$. If $|\gamma| \leqslant 1$, we use the fact that

$$
|\rho(2-\rho)| \geqslant \beta(2-\beta)>\frac{1}{5.5 \log k},
$$

since $k$ is not exceptional. It follows from (2.16) that

$$
\begin{aligned}
\sum_{\substack{\rho \in z\left(x_{1}\right) \\
|\gamma| \leqslant 1}} \frac{2}{|\rho(2-\rho)|} & <11 \log k N(1) \\
& <11\left(\frac{1}{\pi}+C_{1}\right) \log ^{2} k+11\left(C_{2}-\frac{1}{\pi} \log 2 \pi e\right) \log k .
\end{aligned}
$$

If $|\gamma|>1$, we use the estimate $|\rho(2-\rho)| \geqslant \gamma^{2}$, integrate by parts, and use (2.16) to obtain

$$
\begin{aligned}
\sum_{\substack{\rho \in z\left(x_{1}\right) \\
|\gamma|>1}} \frac{2}{|\rho(2-\rho)|} & <2 \int_{1}^{\infty} \frac{d N(t)}{t^{2}}<4 \int_{1}^{\infty} \frac{N(t)}{t^{3}} d t \\
& <\left(\frac{4}{\pi}+2 C_{1}\right) \log k-\frac{4}{\pi} \log 2 \pi+C_{1}+2 C_{2} .
\end{aligned}
$$

The lemma then follows from (3.14), (3.16), (3.17), and the fact that $\left|\zeta^{\prime}(2) / \zeta(2)\right|<.57$.

THEOREM 3.6. If $m$ is a positive integer, $x>2,0<\delta<(x-2) /(m x), H \geqslant 1$, and $A_{m}(\delta)=\delta^{-m} \sum_{j=0}^{m}\left(\begin{array}{c}m \\ j\end{array}\right)(1+j \delta)^{m+1}$, then

$$
\begin{aligned}
\frac{\varphi(k)}{x}\left|\psi(x ; k, l)-\frac{x}{\varphi(k)}\right|< & \left(1+\frac{m \delta}{2}\right) \sum_{\substack { x \\
\begin{subarray}{c}{\rho \in z(x) \\
|\gamma| \leqslant H{ x \\
\begin{subarray} { c } { \rho \in z ( x ) \\
| \gamma | \leqslant H } }\end{subarray}} \frac{x^{\beta-1}}{|\rho|}+\frac{m \delta}{2} \\
& +A_{m}(\delta) \sum_{\substack { x \\
\begin{subarray}{c}{\rho \in z(x) \\
|\gamma|>H{ x \\
\begin{subarray} { c } { \rho \in z ( x ) \\
| \gamma | > H } }\end{subarray}} \frac{x^{\beta-1}}{|\rho(\rho+1) \cdots(\rho+m)|}+\varepsilon_{1},
\end{aligned}
$$

where

$$
\varepsilon_{1}=\frac{k}{x}\left[\frac{\log k \log x}{\log 2}+\frac{k}{4} \log k+C_{3} \log { }^{2} k+\left(C_{4}+1\right) \log k+C_{5}+1\right] .
$$

Proof. From Lemma 3.2 we obtain

$$
\begin{aligned}
\left|E_{m}(x, \pm \delta x)\right| \leqslant & \frac{1}{\varphi(k)} \sum_{x} \sum_{\rho \in z(x)}\left|\frac{\sum_{j=0}^{m}\left(\begin{array}{c}
m \\
j
\end{array}\right)(-1)^{m+j+1}(x \pm j \delta x)^{m+\rho}}{\rho(\rho+1) \cdots(\rho+m)}\right| \\
& +\left|\int_{0}^{ \pm \delta x} \cdots \int_{0}^{ \pm \delta x} f\left(x+y_{1} \cdots+y_{m}\right) d y_{1} \cdots d y_{m}\right| .
\end{aligned}
$$

For the zeros with $|\gamma|>H$ the summand is bounded in absolute value by

$$
\frac{x^{\beta+m}}{|\rho(\rho+1) \cdots(\rho+m)|} \sum_{j=0}^{m}\left(\begin{array}{c}
m \\
j
\end{array}\right)(1+j \delta)^{m+1} .
$$

For the zeros with $|\gamma| \leqslant H$, we write the summand inside the absolute values as

$$
\frac{x^{\rho+m}}{\rho} \int_{0}^{ \pm \delta} \cdots \int_{0}^{ \pm \delta}\left(1+y_{1}+\cdots+y_{m}\right)^{\rho} d y_{1} \cdots d y_{m} \text {. }
$$


The integrand satisfies

$$
\left|\left(1+y_{1}+\cdots+y_{m}\right)^{\rho}\right| \leqslant 1+\sum_{j=1}^{m}\left|y_{j}\right|
$$

so that the absolute value of the summand does not exceed

$$
\frac{x^{\beta+m}}{|\rho|} \int_{0}^{\delta} \cdots \int_{0}^{\delta}\left(1+y_{1}+\cdots+y_{m}\right) d y_{1} \cdots d y_{m}=\frac{x^{\beta+m}}{|\rho|} \delta^{m}\left(1+\frac{m \delta}{2}\right) .
$$

If $y>1$, then from (3.10) we obtain

$$
|f(y)| \leqslant \frac{1}{2} \sum_{n=1}^{\infty} \frac{y^{-n}}{n}+\left|d_{2}\right| \log y+\left|d_{1}+d_{2}\right|
$$

since $a=0$ for half of the characters and $a=1$ for the rest. Hence for $\left|y_{i}\right| \leqslant \delta x$ and $0<\delta<(x-2) /(m x)$ we have

$$
\left|f\left(x+y_{1}+\cdots+y_{m}\right)\right|<\frac{1}{2} \log 2+\left|d_{2}\right| \log 2 x+\left|d_{1}+d_{2}\right| .
$$

From (3.1) and Lemma 3.5 we obtain

$$
\begin{aligned}
& \int_{0}^{ \pm \delta x} \cdots \int_{0}^{ \pm \delta x} f\left(x+y_{1}+\cdots+y_{m}\right) d y_{1} \cdots d y_{m} \\
& \quad<(\delta x)^{m}\left[\frac{1}{2} \log 2+\frac{\log k}{\log 2} \log 2 x+\frac{k}{4} \log k+C_{3} \log ^{2} k+C_{4} \log k+C_{5}\right]
\end{aligned}
$$

The result then follows from Lemma 3.4, (3.18), (3.19), (3.20), and (3.21).

In order to simplify the statements of results, we shall use the notation $L=\log k$ and $H=k^{\alpha}$. As in Rosser and Schoenfeld [10], we use

and also

$$
K_{n}(z, y)=\frac{1}{2} \int_{y}^{\infty} u^{n-1} \exp \left[-\frac{z}{2}\left(u+\frac{1}{u}\right)\right] d u
$$

$$
\varphi_{n}(t)=\frac{x^{-1 / R \log k t}}{t^{n+1}}
$$

LEMMA 3.7. If $k$ is not an exceptional modulus, $k \geqslant 10, x \geqslant \exp \left(\lambda R L^{2}\right)$, and $\lambda \geqslant(1+\alpha)^{2}$, then

where

$$
\sum_{\substack{\rho \in z(x) \\|\gamma| \leqslant H}} \frac{x^{\beta-1}}{|\rho|}<\varepsilon_{2}+\varepsilon_{3}+\varepsilon_{4}
$$

$$
\begin{aligned}
\varepsilon_{2}= & \frac{1}{2} x^{-1 / 2}\left\{\frac{1+4 \alpha+\alpha^{2}}{2 \pi} L^{2}+\frac{2+\alpha}{\pi} L+\frac{R(H)}{H}+2 R(1)+C_{1}\right\} \\
& +x^{-1}\left\{k L+\alpha L^{2}\right\}, \\
& \varepsilon_{3}=\varphi_{0}(H) R(H),
\end{aligned}
$$

and

$$
\begin{aligned}
\varepsilon_{4}=\frac{\lambda L^{2}}{2 \pi}\left\{\lambda L^{2}\left[\Gamma\left(-2, \frac{\lambda}{1+\alpha} L\right)-\Gamma(-2, \lambda L)\right]\right. \\
\left.-\log 2 \pi\left[\Gamma\left(-1, \frac{\lambda}{1+\alpha} L\right)-\Gamma(-1, \lambda L)\right]\right\}
\end{aligned}
$$


Proof. Consider first the contribution from the zeros with $\beta=0$. These zeros arise from the factors $1-\chi_{1}(p) p^{-s}$ in (2.1). Let $N_{p}(T)$ be the number of zeros of $1-\chi_{1}(p) p^{-s}$ in the region $|s| \leqslant T$. An elementary argument yields the estimate

$$
N_{p}(T) \leqslant \frac{T \log p}{\pi}+2
$$

Furthermore for each $p$ there exists at most a single zero $\rho \neq 0$ within $\pi / \log p$ of the origin, and for this zero we have $|\rho|>2 \pi / k_{1} \log p$. It follows from (3.25) that

$$
\sum_{\substack{\rho \in z(\chi) \\ \beta=0 \\|\gamma| \leqslant 1}} \frac{1}{|\rho|}<\sum_{p \mid k}\left[\frac{k_{1} \log p}{2 \pi}+\frac{\log p}{\pi}\left(\frac{\log p}{\pi}+2\right)\right]<\frac{k L}{2 \pi}+\frac{L^{2}}{\pi^{2}}+\frac{2 L}{\pi} .
$$

From (3.25) we obtain

$$
\begin{aligned}
\sum_{\substack{\rho \in z(x) \\
\beta=0 \\
1<|\gamma| \leqslant H}} \frac{1}{|\rho|} & =\sum_{\substack{\rho \mid k \\
p+k,}} \int_{1}^{H} \frac{d N_{p}(t)}{t} \\
& \leqslant \sum_{p \mid k}\left[\frac{N_{p}(H)}{H}+\int_{1}^{H} \frac{N_{p}(t)}{t^{2}} d t\right]<\frac{1}{\pi}(1+\log H) L .
\end{aligned}
$$

It follows from (3.26) that

$$
\sum_{\substack{\rho \in z(x) \\ \beta=0 \\|\gamma| \leqslant H}} \frac{1}{|\rho|}<\frac{k}{2 \pi} L+\frac{L^{2}}{\pi^{2}}+\frac{3 L}{\pi}+\frac{L}{\pi} \log H<k L+L \log H,
$$

since $k \geqslant 10$.

The zeros of $L(s, \chi)$ with $\beta>0$ are symmetrically located with respect to the line $\sigma=\frac{1}{2}$. Hence

$$
\sum_{\substack{\rho \in z\left(x_{1}\right) \\|\gamma| \leqslant 1}} \frac{x^{\beta-1}}{|\rho|} \leqslant \sum_{\substack{\rho \in z\left(x_{1}\right) \\|\gamma| \leqslant 1}} \frac{1}{2}\left(\frac{x^{\beta-1}}{\beta}+\frac{x^{-\beta}}{1-\beta}\right)
$$

By Theorem 1.1 and the fact that $k$ is not exceptional we have $\beta>(\log x)^{-1}$, so that $x^{\beta} / \beta$ is an increasing function of $\beta$. It follows that

$$
\begin{aligned}
\sum_{\substack{\rho \in z\left(x_{1}\right) \\
|\gamma| \leqslant 1}} \frac{x^{\beta-1}}{|\rho|} & <\frac{1}{2} N(1)\left(\frac{x^{-1 / R L}}{1-1 / R L}+\frac{x^{-1 / 2}}{\frac{1}{2}}\right) \\
& =\frac{1}{2} N(1) \varphi_{0}(1) \frac{R L}{R L-1}+N(1) x^{-1 / 2} .
\end{aligned}
$$

For the zeros with $1<|\gamma| \leqslant H$ Theorem 1.1 yields

$$
\sum_{\substack{\rho \in z\left(x_{1}\right) \\ 1<|\gamma| \leqslant H}} \frac{x^{\beta-1}}{|\rho|}<\frac{1}{2} \int_{1}^{H} \varphi_{0}(t) d N(t)+\frac{1}{2} \int_{1}^{H} \frac{x^{-1 / 2}}{t} d N(t) .
$$


In the second integral we integrate by parts and apply (2.16) to obtain

$$
\begin{aligned}
\int_{1}^{H} \frac{d N(t)}{t}< & \frac{N(H)}{H}-N(1)+\frac{F(H) \log H}{H}-\frac{1}{2 \pi} \log ^{2} H \\
& -\frac{R(H)}{H}+R(1)+C_{1}\left(1-\frac{1}{H}\right) \\
& <\frac{N(H)}{H}-N(1)+\frac{1}{2 \pi} \log ^{2} H+F(1) \log H+R(1)+C_{1} .
\end{aligned}
$$

Integration by parts yields

$$
\begin{aligned}
\frac{1}{2} \int_{1}^{H} \varphi_{0}(t) d N(t) \\
=\frac{1}{2} \int_{1}^{H} \varphi_{0}(t) d F_{1}(t)+\frac{1}{2} \int_{1}^{H} \varphi_{0}(t) d\left[N(t)-F_{1}(t)\right] \\
=\frac{1}{2} \int_{1}^{H} \varphi_{0}(t) d F_{1}(t)+\frac{1}{2} \varphi_{0}(H)\left[N(H)-F_{1}(H)\right] \\
\quad-\frac{1}{2} \varphi_{0}(1)\left[N(1)-F_{1}(1)\right]-\frac{1}{2} \int_{1}^{H}[N(t)-F(t)] \varphi_{0}^{\prime}(t) d t .
\end{aligned}
$$

The condition $\lambda \geqslant(1+\alpha)^{2}$ implies that $\varphi_{0}^{\prime}(t)>0$ for $1 \leqslant t<H$, and (2.16) yields

$$
\begin{aligned}
-\frac{1}{2} \int_{1}^{H} & {\left[N(t)-F_{1}(t)\right] \varphi_{0}^{\prime}(t) d t } \\
& <\frac{1}{2} \int_{1}^{H} R_{1}(t) \varphi_{0}^{\prime}(t) d t<\frac{1}{2} R(H) \int_{1}^{H} \varphi_{0}^{\prime}(t) d t \\
& =\frac{1}{2} R(H)\left[\varphi_{0}(H)-\varphi_{0}(1)\right] .
\end{aligned}
$$

It follows from (3.28), (3,29), (3.30), and (3.31) that

$$
\begin{aligned}
& \sum_{\substack{\rho \in i\left(x_{1}\right) \\
|\gamma|<H}} \frac{x^{\beta-1}}{|\rho|} \\
& \quad<\frac{1}{2} x^{-1 / 2}\left\{\frac{N(H)}{H}+N(1)+\frac{1}{2 \pi} \log ^{2} H+F(1) \log H+R(1)+C_{1}\right\} \\
& \quad+\frac{1}{2} \int_{1}^{H} \varphi_{0}(t) d F(t)+\varphi_{0}(H) R(H) \\
& \quad+\frac{1}{2} \varphi_{0}(1)\left[\frac{N(1)}{R L-1}+F(1)-R(H)\right] .
\end{aligned}
$$

From (2.16) and the trivial estimate $F(t)<(t \log k t) / \pi$ we obtain

$$
\begin{aligned}
\frac{N(H)}{H}+N(1)+ & \frac{1}{2 \pi} \log ^{2} H+F(1) \log H+R(1)+C_{1} \\
& <\frac{1+4 \alpha+\alpha^{2}}{2 \pi} L^{2}+\frac{2+\alpha}{\pi} L+\frac{R(H)}{H}+2 R(1)+C_{1} .
\end{aligned}
$$


Furthermore we have

$$
\begin{aligned}
\frac{N(1)}{R L-1}+F(1)-R(H) & <F(1) \frac{R L}{R L-1}+\frac{R(1)}{R L-1}-R(1) \\
& <\frac{R L^{2}}{\pi(R L-1)}-C_{1} \frac{R L-2}{R L-1} L<0,
\end{aligned}
$$

since $L \geqslant \log 10$ and $C_{1}>1 / \pi \log 2$. The lemma then follows from (3.27), (3.32), (3.33) and the fact that

$$
\frac{1}{2} \int_{1}^{H} \varphi_{0}(t) d F(t)=\varepsilon_{4}
$$

LEMMA 3.8. If $k \geqslant 10, x \geqslant \exp \left(\lambda R L^{2}\right)$, and $\lambda \leqslant(m+1)(1+\alpha)^{2}$, then

$$
\sum_{\substack{\rho \in z\left(x_{1}\right) \\|\gamma|>H}} \frac{x^{\beta-1}}{|\rho(\rho+1) \cdots(\rho+m)|}<\varepsilon_{5}+\varepsilon_{6}+\varepsilon_{7},
$$

where

$$
\begin{aligned}
\varepsilon_{5}=\frac{x^{-1 / 2}}{2 H^{m+1}} & \left\{\frac{H}{\pi m}(1+\alpha) L+2 R(H)+\frac{C_{1}}{m+1}\right\}+\frac{4 L}{x H^{m}} \\
\varepsilon_{6}=k^{m} L\{ & \frac{\lambda L}{\pi m} K_{2}\left(2 \sqrt{\lambda m} L,(1+\alpha) \sqrt{\frac{m}{\lambda}}\right) \\
& -\sqrt{\frac{\lambda}{m}} \frac{\log 2 \pi}{\pi} K_{1}\left(2 \sqrt{\lambda m} L,(1+\alpha) \sqrt{\frac{m}{\lambda}}\right) \\
& \left.+C_{1} k \sqrt{\frac{\lambda}{m+1}} K_{1}\left(2 \sqrt{(m+1) \lambda} L,(1+\alpha) \sqrt{\frac{m+1}{\lambda}}\right)\right\}
\end{aligned}
$$

and

$$
\varepsilon_{7}=R(H) \varphi_{m}(H) .
$$

Proof. From (3.25) and integration by parts we obtain

$$
\begin{aligned}
\sum_{\substack{\beta \in z(\chi) \\
\beta=0 \\
|\gamma|>H}} \frac{1}{|\gamma|^{m+1}} & =\sum_{\substack{p \mid k \\
p \nmid k_{1}}} \int_{H}^{\infty} \frac{d N_{p}(t)}{t^{m+1}} \\
& \leqslant \sum_{p \mid k}(m+1) \int_{H}^{\infty} \frac{N_{p}(t)}{t^{m+2}} d t \leqslant \sum_{p \mid k}\left[\frac{m+1}{\pi m H^{m}} \log p+\frac{2}{H^{m+1}}\right] \\
& \leqslant \frac{L}{H^{m}}\left[\frac{m+1}{\pi m}+\frac{2}{H \log 2}\right] \leqslant \frac{4 L}{H^{m}} .
\end{aligned}
$$


For the zeros with $\beta>0$ we use Theorem 1.1 and the symmetric location of the zeros with respect to the line $\sigma=\frac{1}{2}$ to obtain

$$
\begin{aligned}
\sum_{\substack{\rho \in z\left(x_{1}\right) \\
|\gamma|>H}} \frac{x^{\beta-1}}{|\rho(\rho+1) \cdots(\rho+m)|} \\
\quad<\frac{1}{2} \int_{H}^{\infty} \varphi_{m}(t) d N(t)+\frac{1}{2} \int_{H}^{\infty} \frac{x^{-1 / 2}}{t^{m+1}} d N(t) .
\end{aligned}
$$

If we integrate by parts in the second integral and apply (2.16), we find that

$$
\begin{aligned}
\int_{H}^{\infty} \frac{d N(t)}{t^{m+1}}< & \frac{-N(H)}{H^{m+1}} \\
& +\frac{1}{H^{m+1}}\left\{\frac{m+1}{m} F_{1}(H)+\frac{m+1}{\pi m^{2}} H+R_{1}(H)+\frac{C_{1}}{m+1}\right\} \\
& <\frac{1}{H^{m+1}}\left\{\frac{1}{m} F(H)+\frac{m+1}{\pi m^{2}} H+2 R(H)+\frac{C_{1}}{m+1}\right\} \\
& <\frac{1}{H^{m+1}}\left\{\frac{H}{\pi m} \log k H+2 R(H)+\frac{C_{1}}{m+1}\right\} .
\end{aligned}
$$

For the first integral we proceed as in (3.31) to obtain

$$
\begin{aligned}
\frac{1}{2} \int_{H}^{\infty} \varphi_{m}(t) d N(t)= & \frac{1}{2} \int_{H}^{\infty} \varphi_{m}(t) d F_{1}(t)+\frac{1}{2} \varphi_{m}(H)\left[F_{1}(H)-N(H)\right] \\
& +\frac{1}{2} \int_{H}^{\infty}\left[F_{1}(t)-N(t)\right] \varphi_{m}^{\prime}(t) d t
\end{aligned}
$$

The condition $\lambda \leqslant(m+1)(1+\alpha)^{2}$ implies that $\varphi_{m}^{\prime}(t)<0$ for $t>H$, so we apply (2.16) and integrate by parts again to obtain

$$
\begin{aligned}
\frac{1}{2} \int_{H}^{\infty} \varphi_{m}(t) d N(t)< & \frac{1}{2} \int_{H}^{\infty} \varphi_{m}(t) d F(t)+\varphi_{m}(H) R(H) \\
& +\frac{C_{1}}{2} \int_{H}^{\infty} \varphi_{m+1}(t) d t=\varepsilon_{6}+\varepsilon_{7}
\end{aligned}
$$

The lemma then follows from (3.38), (3.39), and (3.40).

THEOREM 3.9. Let $k$ be a nonexceptional modulus, $(k, l)=1, k \geqslant k_{0} \geqslant 10$, $m$ be a positive integer, $0<\delta<(x-2) /(m x)$, and $x \geqslant \exp \left(\lambda R L^{2}\right)$. Let $(1+\alpha)^{2} \leqslant \lambda \leqslant$ $(m+1)(1+\alpha)^{2}$ and

$$
L>\max \left\{\frac{2+2 \alpha}{\lambda-1-\alpha}+\frac{\log 2 \pi}{1+\alpha}, \frac{2}{\lambda-1}+\log 2 \pi\right\} .
$$

If $\lambda>m(1+\alpha)^{2}$, then let

$$
L>\frac{2}{2 \sqrt{m \lambda}-m-1}+\frac{\log 2 \pi}{1+\alpha} .
$$


Then

$$
\begin{aligned}
\frac{\varphi(k)}{x} \mid \psi(x ; & k, l)-\frac{x}{\varphi(k)} \mid \\
< & \left(1+\frac{m \delta}{2}\right) k_{0}\left[\varepsilon_{2}\left(k_{0}\right)+\varepsilon_{3}\left(k_{0}\right)+\varepsilon_{4}\left(k_{0}\right)\right]+\frac{m \delta}{2} \\
& +\varepsilon_{1}\left(k_{0}\right)+A_{m}(\delta) k_{0}\left[\varepsilon_{5}\left(k_{0}\right)+\varepsilon_{6}\left(k_{0}\right)+\varepsilon_{7}\left(k_{0}\right)\right] .
\end{aligned}
$$

Proof. We may assume that $x=\exp \left(\lambda R L^{2}\right)$, since our upper bound from Theorem 3.6 is decreasing in $x$. By Theorem 3.6 and Lemmas 3.7 and 3.8 it suffices to prove that (for fixed $\lambda, \eta, m$, and $\alpha) \varepsilon_{1}(k)$ and $k \varepsilon_{i}(k), i=2, \ldots, 7$, are decreasing in $k$. Of these, the functions $\varepsilon_{1}, k \varepsilon_{2}$, and $k \varepsilon_{5}$ are easily shown to be decreasing in $L$.

It follows from (3.41) that $L \exp [L(1-\lambda /(1+\alpha)-\alpha)]$ is decreasing in $L$, and this suffices to prove that $k \varepsilon_{3}$ and $k \varepsilon_{7}$ are decreasing in $L$.

From (3.24) we obtain

$$
2 \pi k \varepsilon_{4}(k)=\int_{1}^{1+\alpha} f(L, u) d u
$$

where

$$
f(L, u)=\left(L^{2} u-L \log 2 \pi\right) \exp \left[\left(1-\frac{\lambda}{u}\right) L\right] .
$$

Note that for $1 \leqslant u \leqslant 1+\alpha$ we have

$$
\begin{aligned}
L^{-1} \exp \left[\left(\frac{\lambda}{u}\right.\right. & -1) L] \frac{d}{d L} f(L, u) \\
& <(L+2) u+\frac{\lambda \log 2 \pi}{u}-\log 2 \pi-\lambda L \\
& <\max \left\{(L+2)(1+\alpha)+\frac{\lambda \log 2 \pi}{1+\alpha}, L+2+\lambda \log 2 \pi\right\} \\
& -\log 2 \pi-\lambda L<0
\end{aligned}
$$

by (3.41). Hence $k \varepsilon_{4}$ is decreasing in $L$.

From (3.36) we obtain

$$
2 k \varepsilon_{6}(k)=\frac{1}{\pi} \int_{1+\alpha}^{\infty} g_{1}(L, u) d u+C_{1} \int_{1+\alpha}^{\infty} g_{2}(L, u) d u
$$

where

$$
\begin{aligned}
& g_{1}(L, u)=\left(L^{2} u-L \log 2 \pi\right) \exp \left[L\left(m+1-m u-\frac{\lambda}{u}\right)\right], \\
& g_{2}(L, u)=L \exp \left\{L\left[m+2-(m+1) u-\frac{\lambda}{u}\right]\right\}
\end{aligned}
$$

The first integrand satisfies

$$
\begin{aligned}
\frac{d}{d L} g_{1}(L, u)< & L\left\{(L u-\log 2 \pi)\left(m+1-m u-\frac{\lambda}{u}\right)+2 u\right\} \\
& \times \exp \left[L\left(m+1-m u-\frac{\lambda}{u}\right)\right]<0,
\end{aligned}
$$


provided

$$
L>\frac{2}{\lambda / u+m u-m-1}+\frac{\log 2 \pi}{1+\alpha}, \quad u \geqslant 1+\alpha .
$$

If $1+\alpha<\sqrt{\lambda / m}$, this condition is met by (3.42) and if $1+\alpha \geqslant \sqrt{\lambda / m}$, then it follows from (3.41).

The second integral in (3.44) can similarly be shown to be decreasing provided $L>(\lambda /(1+\alpha)+(m+1) \alpha-1)^{-1}$.

4. Computations. In this section we describe the methods used in the preparation of Table 1. Note that Theorem 1.2 gives estimates for $\theta(x ; k, l)$ as well as $\psi(x ; k, l)$. By a result of Schoenfeld [11], we have

$$
\begin{aligned}
0 & \leqslant \psi(x ; k, l)-\theta(x ; k, l) \leqslant \psi(x ; 1,1)-\theta(x ; 1,1) \\
& <1.001093 x^{1 / 2}+3 x^{1 / 3} .
\end{aligned}
$$

Hence we obtain the estimate

$$
\left|\theta(x ; k, l)-\frac{x}{\varphi(k)}\right|<1.001093 x^{1 / 2}+3 x^{1 / 3}+\left|\psi(x ; k . l)-\frac{x}{\varphi(k)}\right| .
$$

and the extra terms are negligible for the range of $x$ under consideration.

Estimates for the incomplete gamma function and incomplete Bessel functions may be found in [11] and [10]. Upper bounds for $K_{\nu}(z, x)$ are provided by Lemma 4. Lemma 5, (2.30), and (2.31) of [10]. In addition, if $x<1$, we can use Lemma 3 combined with Lemma 4 and the asymptotic expansions of $K_{\nu}(z)(9.7 .2$ of [1]). A lower bound for $K_{1}(z, x)$ is provided by Lemma 4 or (2.22) and (2.33) of [10], resulting in the estimate

$$
\begin{aligned}
K_{1}(z, x)>\frac{e^{-z}}{2 z}\{[1 & \left.+\frac{\sqrt{2}}{8} y\left(3-y^{2}-\frac{3}{2 z}\right)\right] e^{-z^{2}} \\
& \left.+\left(z+\frac{3}{8}-\frac{3}{16 z}\right) \sqrt{2} \int_{1}^{x} e^{-z n^{2}} d w\right\}
\end{aligned}
$$

If $x<1$, another method for bounding $K_{1}(z, x)$ from below is to use (2.10) of [10] and 9.7.2 of [1]. Other methods for estimating $K_{\nu}(z, x)$ are available in [10] and [12], but in the interests of simplifying the computations these were not used in the preparation of Table 1.

The choices of the parameters $m, \eta, \alpha$, and $\delta$ are completely at our disposal. We used $m=2$ since it seemed to give the best results. Tables 2 and 3 give the values of $\eta$ and $\alpha$ used in the preparation of Table 1. The best values of $\alpha$ turn out to be only slightly less than $\sqrt{\lambda}-1$, and the choice $\alpha=\sqrt{\lambda}-1$ would lead to results that are nearly as good. The major effect of $\eta$ is to control the size of $\varepsilon_{3}$ and $\varepsilon_{7}$. For this reason, and the fact that the best $\alpha$ is near $\sqrt{\lambda}-1$, we chose $\eta$ to minimize $R\left(k^{\sqrt{\lambda}-1}\right)$.

This leaves only $\delta$ to be chosen. For $m=2$, the optimal $\delta$ is approximately that which minimizes

$$
\delta\left(1+w_{1}+10 w_{2}\right)+\left(4 \delta^{-2}+12 \delta^{-1}+18\right) w_{2},
$$


where

$$
w_{1}=k \varepsilon_{2}+k \varepsilon_{3}+k \varepsilon_{4}, \quad w_{2}=k \varepsilon_{5}+k \varepsilon_{6}+k \varepsilon_{7} .
$$

We can then find $\delta$ by elementary calculus. If $1+w_{1}<102 w_{2}$, a minimum exists at the positive real root of $\delta^{3}-a \delta-2 a / 3$, where $a=12 w_{2} /\left(1+w_{1}+10 w_{2}\right)$. This leads to the choice

$$
\delta=D+a /(3 D)
$$

where

$$
D=\left[\frac{a}{3}\left(1+\sqrt{1-\frac{a}{3}}\right)\right]^{1 / 3} .
$$

All computations were performed on the CDC Cyber Computer at Michigan State University, using double precision Fortran (approximately 28 significant decimal digits). We have listed in Table 1 only values of $c$ for which we were able to find appropriate values of $\eta$ and $\alpha$, but Theorem 3.9 may actually yield slightly smaller values of $c$.

\begin{tabular}{|c|c|c|c|c|c|c|c|c|c|c|}
\hline$b$ & 1 & .5 & .2 & .1 & .05 & .01 & .005 & .001 & .0001 & .00001 \\
\hline 1 & .500 & .500 & .500 & .500 & .500 & .500 & .500 & .436 & .369 & .321 \\
\hline 2 & .500 & .500 & .500 & .500 & .497 & .435 & .413 & .369 & .321 & .285 \\
\hline 3 & .495 & .476 & .451 & .430 & .410 & .369 & .352 & .321 & .285 & .255 \\
\hline$\therefore$ & .411 & .398 & .379 & .365 & .351 & .320 & .308 & .285 & .255 & .233 \\
\hline 5 & .352 & .342 & .329 & .318 & .307 & .284 & .275 & .255 & .233 & $.2 ! 3$ \\
\hline t. & .308 & .301 & .291 & .283 & .274 & .255 & .249 & .233 & .213 & .198 \\
\hline$i$ & .275 & .269 & .261 & .254 & .248 & .233 & .226 & .213 & .198 & .183 \\
\hline 8 & .249 & .244 & .237 & .232 & .226 & $.21,3$ & .208 & .198 & .183 & .171 \\
\hline 3 & .227 & .223 & .217 & .212 & .208 & .197 & .193 & .183 & .171 & .162 \\
\hline !6 & .208 & .206 & .201 & .197 & .193 & .183 & .179 & .171 & .162 & .152 \\
\hline 11 & .194 & .191 & .186 & .183 & .179 & .171 & .158 & .162 & .152 & .144 \\
\hline 12. & .180 & .177 & .174 & .171 & .168 & .161 & .159 & .152 & .144 & .136 \\
\hline 13 & .168 & .166 & .164 & .161 & .158 & .152 & .149 & .144 & .136 & .129 \\
\hline 14 & .159 & .157 & .154 & .152 & .149 & .144 & .141 & .136 & .129 & .124 \\
\hline 15 & .150 & .148 & .145 & .143 & .141 & .136 & .134 & .129 & .124 & .119 \\
\hline 20 & .117 & .116 & .115 & .113 & .112 & .109 & .108 & .105 & .101 & .097 \\
\hline 25 & .096 & .095 & .094 & .093 & .092 & .090 & .089 & .087 & .085 & .083 \\
\hline 30 & .082 & .082 & .081 & .080 & .080 & .078 & .077 & .076 & .074 & .072 \\
\hline 35 & .071 & .071 & .070 & .070 & .069 & .048 & .068 & .067 & .065 & .063 \\
\hline 40 & .063 & .063 & .062 & .062 & .061 & .061 & .060 & .059 & .058 & .057 \\
\hline 45 & .056 & .056 & .056 & .055 & .055 & .054 & .054 & .053 & .052 & .051 \\
\hline 50 & .051 & .051 & .050 & .050 & .050 & .049 & .040 & .048 & .048 & .047 \\
\hline ho & .043 & .043 & .042 & .042 & .042 & .042 & .041 & .041 & .041 & .041 \\
\hline$i 0$ & .038 & .038 & .038 & .037 & .037 & .037 & .037 & .036 & .036 & .036 \\
\hline 80 & .033 & .033 & .033 & .033 & .033 & .033 & .033 & .032 & .032 & .032 \\
\hline 30 & .030 & .030 & .030 & .030 & .029 & .029 & .029 & .029 & .029 & .028 \\
\hline 100 & .027 & .027 & .027 & .027 & .027 & .026 & .026 & .026 & .026 & .026 \\
\hline
\end{tabular}

TABLE 2

$\eta$ 
TABLE 3

$\alpha$

\begin{tabular}{|c|c|c|c|c|c|c|c|c|c|c|}
\hline b & 1 & .5 & .2 & .1 & .05 & .01 & .005 & .001 & .0001 & .00001 \\
\hline 1 & .879 & 1.06 & 1.345 & 1.599 & 1.873 & 2.564 & 2.879 & 3.584 & 4.584 & 5.584 \\
\hline$\hat{\imath}$ & .461 & .552 & .700 & .829 & .969 & 1.296 & 1.451 & 1.791 & 2.281 & 2.786 \\
\hline 3 & .319 & .383 & .484 & .564 & .647 & .851 & .953 & 1.187 & 1.52 .4 & 1.858 \\
\hline 4 & .246 & .294 & .369 & .434 & .480 & .644 & .717 & .892 & 1.143 & $1.39 \%$ \\
\hline ; & .202 & .242 & .298 & .338 & .391 & .522 & .579 & .715 & .915 & 1.114 \\
\hline 5 & .170 & .207 & .253 & .286 & .331 & .431 & .488 & . 599 & .762 & .932 \\
\hline 7 & .149 & .177 & .214 & .233 & .287 & .380 & .412 & .316 & .653 & .794 \\
\hline 8 & .134 & .153 & .177 & .207 & .238 & .323 & .358 & .445 & .574 & .694 \\
\hline 9 & .123 & .129 & .160 & .186 & $.2 \mathrm{i} 3$ & .281 & .332 & .406 & .512 & .617 \\
\hline in & .106 & .118 & .146 & .169 & .195 & .254 & .281 & .367 & .463 & .957 \\
\hline 11 & .098 & .116 & .134 & .154 & $.17 \varepsilon$ & .232 & .264 & .335 & .421 & .508 \\
\hline 12 & .085 & .101 & .124 & .143 & .164 & $.21 \%$ & .236 & .288 & .388 & .466 \\
\hline 13 & .085 & .096 & .115 & .133 & .153 & .199 & .219 & .275 & .360 & .432 \\
\hline 14 & .075 & .089 & .108 & .125 & .143 & .186 & .204 & .249 & .326 & .402 \\
\hline 15 & .070 & .083 & .101 & .117 & .134 & .174 & .191 & .232 & .302 & .375 \\
\hline$\therefore 0$ & .048 & .064 & .067 & .091 & .089 & .133 & .147 & .177 & .228 & .285 \\
\hline 25 & .045 & .046 & .055 & .063 & .072 & .108 & .119 & .143 & .178 & .228 \\
\hline 3i) & .033 & .039 & $.04 i$ & .054 & .051 & .091 & .099 & .120 & .150 & .185 \\
\hline 35 & .030 & .033 & .041 & .047 & .053 & .072 & .082 & .104 & .129 & .154 \\
\hline 40 & .026 & .030 & .036 & .042 & $.04 i$ & $.0 n 3$ & .076 & .091 & .113 & .135 \\
\hline 4.5 & .024 & .027 & .033 & .037 & .042 & .06 .2 & .058 & .081 & .101 & .120 \\
\hline 50 & .022 & .025 & .030 & .034 & .038 & .048 & .058 & .074 & .091 & .109 \\
\hline 60 & .018 & .021 & .025 & .029 & .033 & .041 & $.0 \div 5$ & .062 & .077 & .091 \\
\hline 70 & .016 & .018 & .021 & .025 & .028 & .035 & .039 & .046 & .066 & .078 \\
\hline 80 & .015 & .016 & .020 & .021 & .024 & .031 & .034 & .047 & .057 & .069 \\
\hline 90 & .013 & .015 & .017 & .020 & .021 & .028 & .032 & .042 & .052 & .062 \\
\hline 200 & .012 & .013 & .015 & .018 & .020 & .025 & .027 & .033 & .047 & .056 \\
\hline
\end{tabular}

The conditions (3.41) and (3.42) fail to hold for several entries of Table 1, and this required a check of all values of $k$ up to a point where (3.41) and (3.42) were in effect.

This paper is based on the work contained in the author's Ph.D. thesis, written under the direction of Professor Paul T. Bateman at the University of Illinois. The author acknowledges with gratitude many valuable discussions with Professor Bateman.

Department of Mathematics

Michigan State University

East Lansing. Michigan 48824

1. M. Abramowitz \& I. Stegun, editors, Handbook of Mathematical Functions, Dover, New York. 1965.

2. R. BACKLUND, “Über die Nullstellen der Riemannschen Zetafunction," Acta Math., v. 41, 1918, pp. $374-375$.

3. H. DavenPort, Multiplicative Number Theory, Markham, Chicago, 1967.

4. A. E. Ingham, The Distribution of Prime Numbers, Cambridge Univ. Press, London, 1932.

5. K. S. MCCURLEY, “Explicit zero-free regions for Dirichlet $L$-functions.” (To appear.) 
6. F. W. J. Olver, Asymptotics and Special Functions, Academic Press, New York, 1974.

7. K. Prachar, Primzahlverteilung, Springer-Verlag, New York, 1957.

8. H. Rademacher, "On the Phrägmen-Lindelof theorem and some applications," Math. Z., v. 72 , 1959. pp. 192-204.

9. J. B. Rosser, “Explicit bounds for some functions of prime numbers,” Amer. J. Math., v. 63, 1941, pp. 211-232.

10. J. B. Rosser \& L. SChoenfeld, "Sharper bounds for the Chebyshev functions $\theta(x)$ and $\psi(x)$," Math. Comp., v. 29, 1975, pp. 243-269.

11. L. SChoenfeld, "Sharper bounds for the Chebyshev functions $\theta(x)$ and $\psi(x)$. II," Math. Comp., v. 30. 1976, pp. 337-360.

12. R. Terras, “A Miller algorithm for an incomplete Bessel function,” J. Comput. Phys., v. 39, 1981, pp. $233-240$. 